\title{
HISTOPATHOLOGICAL AND SCANNING ELECTRON MICROSCOPE STUDIES OF ENTOMOPATHOGENIC FUNGUS (BEAUVERIA SPP.) ON DIFFERENT STAGES OF MUSCA DOMESTICA (DIPTERA: MUSCIDAE)
}

By

AHMED M. ABDEL-GAWAD ${ }^{1}$, MARWA M. KHALIFA ${ }^{1 *}$, MAHMOUD D. ELHARIR ${ }^{2}$, MAHMOUD A. MAHMOUD ${ }^{3}$ and MENNA A. EL-GENEADY ${ }^{1}$ Department of Parasitology ${ }^{1}$, Department of Microbiology ${ }^{2}$, and Department of Pathology ${ }^{3}$, Faculty of Veterinary Medicine, Cairo University ${ }^{3}$, Giza, Egypt ( ${ }^{*}$ Correspondence: marwaehab40@yahoo.com)

\section{Abstract}

The entomopathogenic fungus, Beauveria spp. was isolated from house flies collected from different localities in various Egyptian Governorates. Flies were trapped from garbage piles, dairy and meat production processes on cattle farms and fowl farms. The study focused on Beauveria spp. as entomopathogens were widely used in recent decades as a biological control of the population of house flies, to avoid hazardous effects of insecticides on man animals, and natural eco-systems. Experimental laboratory trials were done using immersion of larvae on serial dilution of Beauveria spores reared different stages of the house flies. Infected larvae, pupae and adults were examined macroscopically for visible growth of fungi, before further studies using scanning electron microscope and histopathological methods, to investigate destructive impact of the fungus on the external and internal structures. The previous investigations revealed adhesion growth and propagation of the conidia on the cuticle of all stages, including compound eyes, setae of the legs, thorax, wings and abdomen of adults. Penetration and propagation of the conidia was evident in larval internal tissues causing lysis of fat cells, and of thoracic muscles of adults.

Keywords: Egypt, Musca domestica, Entomopathogens, SEM, Histopathology, Biological control.

\section{Introduction}

Since the $20^{\text {th }}$ Century, parasitologists and medical entomologists have recorded the mechanical or biological transmission of different pathogens by Musca domestica or the house fly, Diptera: Muscidae (Farooq and Freed, 2016). House flies act as infective stages of helminthes, protozoan cysts, and bacterial agents which induce fatal diseases such as typhoid, cholera, tuberculosis, bacillary and amebic dysentery, infantile diarrhea and anthrax in man and animals (Lecuona et al, 2005; Förster et al, 2009). In recent decades, research established the serious role of $M$. domestica as transmitters of dangerous fungi types, chiefly Aspergillus spp. (Sales et al, 2002) that induce hazardous diseases such as fungal pneumonia in man and animals and nail infections in humans (Schuster et al, 2002; Amaike et al, 2011). Conventional insecticides are the primary control method of $M$ domestica. However, they have created severe problems such as insecticide resistance in addition to the serious residual effects of chemicals in consumed animal carcasses. Evidence proved that houseflies were resistant to all conventional insecticide groups as organophosphates, organochlorines, carbamates and pyrethroids (Azzam and Hussein, 2002). This problem and insecticide expenses paved the way for other alternatives agents as entomopathogenic fungi as a potential biological control of insect pest line (Geden, 2012). Compared to devastating insecticide effects, entomopathogenic fungi have less hazardous impacts on global eco-systems including mammalian cycles. The presence of entomopathogenic fungi in areas and places where houseflies feed, dwell and propagate provided significant opportunities to use it to manage housefly populations (Khanet al, 2012)

There are many examples of successful fungus-based insect control programs by using Beauveria bassiana and Metarhizium anisopliae (Shah and Pell, 2003; Roberts and Leger, 2004). B. bassiana is the commonest employed fungus for house-fly control with 
high mortality rates within 5-15 days.

The present study aimed to study the role of Beauveria bassiana as a biological control versus different developmental stages of Musca domestica. Stages were immersed in serial dilution of Beauveria spores for certain time, and reared under close observation to evaluate the fungi effect. Infected larvae, pupae and adults were examined macroscopically for fungi growth by SEM and histopathologically for exposed and non-exposed stages, to evaluate fungus destructive effect on external and internal structures in comparison with control non-exposed ones.

\section{Material and Methods}

Collection of Musca domestica: M. domestica as identified (Hafez et al, 1971), were collected from garbage piles, dairy and meat processing plants and fowl farms from the Giza (Nahia and Talbia district), El-Behira, El Gharbia and Kafr El-Sheikh Governorates. About fifty house-flies were gathered from June to September 2019 at each governorate, using a sweeping net.

Rearing: Flies were bred under laboratory conditions of $25-28 \pm 5^{\circ} \mathrm{C} \& 40-65 \% \mathrm{RH}$ in $30 \times 30 \times 30 \mathrm{~cm}$ insect-proof wooden cage. Each has three sides of narrow wire mesh and a fourth side that is vertically movable with a hole in its middle. A piece of cotton cloth was tightly fixed to the hole by adhesive glue. Cloth was closed at its terminal end by a rubber band to enable safe access to the inside of the cage. Adult flies were fed a mixture of equal parts $(25 \mathrm{gm})$ of powdered milk and granulated sugar, mixed with water. A piece of cotton was soaked in the mixture and put in Petri-dishes in breeding cage. Hatched larvae were transferred to $250 \mathrm{ml}$. glass beakers containing a larval diet of 5 gm. wheat bran, $2 \mathrm{gm}$. milk powder and $1 \mathrm{ml}$. honey mixed with $10 \mathrm{ml}$ of water. Diet was replenished daily until the emergence of pupae. Pupae were transferred to $250 \mathrm{ml}$. glass beakers with saw dust in the bottom, covered by a piece of goose, tied by an elastic band, and put in the breeding cage at 25$28 \pm 5^{\circ} \mathrm{C} \& 40-65 \% \mathrm{RH}$.
Isolation of fungi from collected samples.

Ordinary fungal growth media: Collected flies from each locality were separated in five groups. The first group was composed of 30 flies collected from garbage piles in the Nahia District. The second group contained 25 flies from garbage piles in the Talbia district and Giza governorate. Other groups each had fifty flies from a dairy processing plant in El-Behira a fowl farm in El Gharbia and a meat processing plant in Kafr elShikh. All flies were preserved in test tubes and placed in a freezer for 5 minutes to anesthetize them. To sterilize the flies, they were washed in 1\% sodium hypo-chlorite solution and rinsed twice in sterile distilled water for 3 minutes and 1 minute respectively (Seymour et al, 1984). The flies were transferred to a porcelain crucible for maceration, by adding a saline solution drop by drop using smooth grinding. About $0.1 \mathrm{ml}$ of the macerated preparation was spread on a fungal growth media containing $32.5 \mathrm{gm}$. SDA (Sabouraud Dextrose Agar) in $500 \mathrm{ml}$ distilled water with $0.025 \mathrm{gm}$.

Chloramphenicol was used as antibiotic to inhibit bacterial growth and $0.125 \mathrm{gm}$ cycloheximide as saprophytic fungicide. Five plates of the prepared fungal growth media were used for each flies, group. Plates were stored at $28^{\circ} \mathrm{C} \pm 1 \& \mathrm{RH}$ of $\geq 80 \%$. Daily observations for 15 days to observe fungal colony growth. Isolated colony was macroscopically identified by specific color and shape and examined microscopically (Sales et al, 2002).

Growth media for entomopathogenic fungi: To isolate lethal from non-lethal fungi usually present in adult fly bodies, $0.1 \mathrm{ml}$ of the macerated preparation was cultivated into the selective media specific for entomopathogenic fungi, Beauveria and Metarhizium species. Media consisted of $32.5 \mathrm{gm}$ SDA suspension (Sabouraud Dextrose Agar) in $500 \mathrm{ml}$ distilled water with $0.025 \mathrm{gm}$ chloramphenicol, added to $0.1 \mathrm{gm}$ of selective substance, dodine (Zarrin et al, 2007), as an inhibitor to the other saprophytic fungi. 
Infection of $M$. domestica larvae: To infect larvae with the isolated entomopathogenic fungus, spore suspensions were prepared by adding $10 \mathrm{ml}$ of distilled water and $0.05 \mathrm{ml}$ of $0.5 \%$ tween 20 to the fungal colony plates. Fungal spores were counted by a hemocytometer using $10 \mu \mathrm{m}$ of spore suspension (Farooq and Freed, 2016). Serial concentrations of $10^{4}, 10^{5}, 10^{6}, 10^{7}, 10^{8}, 10^{9}, 10^{10}$ spores $/ \mathrm{ml}$ were prepared.

To study cumulative lethal effects of the isolate on different stages of the flies, forty larvae of $2^{\text {nd }}$ stage were infected by dipping them for 10 seconds in each concentration. Three larvae replicas infected by each spore concentration were used. Control groups of larvae were prepared by dipping them in a solution of a $0.05 \%$ Tween 80 only. Larvae were transferred to small plates containing larval diet, and incubated at $25 \pm 5^{\circ} \mathrm{C} \& 65 \%$ $\mathrm{RH}$, for 4 daily observations, to count them. From $5^{\text {th }}$ day onward, larvae were checked daily for pupal emergence, which relocated to a glass beaker containing a layer of sawdust. The emerged adult flies were checked daily for 14 days to count the mortality percentages resulting from the fungi.

Infection of adult M. domestica: Forty flies from fungal spore concentrations of $10^{5}$, $10^{8} \& 10^{10}$ were used for each infection trial. A patch of same flies number was used as a control by immersing them into a solution of $0.05 \%$ Tween 80 only. All infected and control groups were stored and fed as previously described and were observed daily for 14 days for mortality recorded.

The cadaver was daily collected and put on moist filter papers in Petri-dishes to facilitate fungal sporulation (Farooq and Freed, 2016). Mortality resulting from fungal infection was identified by examining the fungal growth color and shape, indicative for each specific type of entomopathogenic fungi (Steinkraus et al, 1990). For more confirmation, the cadaver were washed in $10 \mathrm{ml}$ sterile distilled water, stirred for 30 seconds to extract spores, and examined under a light microscope (Mwamburi et al, 2010).
A histopathological study investigated the entomopathogenic fungus effect on internal and external tissues of the different stages. Five samples from each group of infected larvae and adults with fungal concentration of $10^{10}$ were fixed in $10 \%$ formalin. Then, they were washed in water, dehydrated in ethanol, cleared in xylene and embedded into paraffin wax (Bancroft et al, 1996). By standard histological protocols, sectioning (5-10 $\mu \mathrm{m})$ were put on slides and stained with hematoxylin and eosin (H\&E). Masson's trichrome (MT) and periodic acid Schiff (PAS) were also used (Toledo et al, 2010)

SEM: Samples from different stages of infected and control larvae, pupa and adult with fungal concentration of $10^{10}$ only were fixed in $2.5 \%$ glutaraldehyde of $\mathrm{PH} 7.2$ buffer for $6 \mathrm{hrs}$, and then fixed in $1 \%$ Osmium tetroxide $\left(\mathrm{OsO}_{4}\right)$ for $1 \mathrm{hr}$. Larvae were dehydrated in ascending ethanol series, dried in $\mathrm{CO}_{2}$ critical drier (Autosamdri-815, Germany) and glued over stubs, coated with gold, examined and photographed using SEM (JSM 5200, Electron Microanalyzer, Jeol, Japan) in a SEM Center, Faculty of Agriculture, Cairo University.

\section{Results}

Thorough histopathological investigations were implemented to investigate destructive effects of naturally isolated entomopathogenic fungus, Beauveriaspp on the different stages of $M$. domestica. Two special stains, Masson trichrome (MS) and Periodic Acid Schiff (PAS) stains were used in addition to the H\&E stain. The MS stain functions as an identifier of the internal tissues of dipterous flies. The PAS stain effectively in defines the hyphae, and spores of the fungus, which stained in purple-red, counter stain of the flies' tissues that colored dark blue.

Histopathology of infected larvae: Control larvae stained by the PAS stain showed normal outer structures such as, cuticular integument, muscular system and cephalophryngeal region without any signs of fungal spores and/or hyphae presence. In contrast to the controlled ones, infected larvae stained 
by PAS showed degenerative lesions including lysis of adipose tissue in tbody cavity. Also, stained larvae by PAS and MT showed fungal spores in cuticular integument, beside its penetration of the body cavity.

Histopathology of infected adults: Adults of non-infected stained by PAS showed normal external structures including head, thorax, abdomen and compound eyes. Internal tissues like testes and Malpighian tubules were also intact. But, 24hrs postmortem females by $10^{10}$ concentrated fungal spores stained by MT exhibited aggregations of fungal spores in abdominal region near hindgut and vagina.

The present study showed that over time there was a severe destructive impact of the fungus on the outer and inner structures of adult flies. In $72 \mathrm{hrs}$ postmortem adults stained by MT stain, exhibited muscular lysis in the thoracic region. By using MT stain, fungal were clearly aggregated in the compound eyes. The previous facts worsened in case of 10 days cadavers, where all parts of the outer cuticle include compound eyes, thorax, legs and abdomen were mushroomed by hyphae and fungal spores. In sections those stained by PAS \& MT, the proboscis was attacked by fungal spores, while control cadaver stained by H\&E and MT showed normal legs, muscle and head.

SEM of infected larvae, pupae and adults of $M$. domestica by $10^{10}$ spore concentration: SEM images of $M$. domestica control larvae showed normal mouth parts with intact oral hooks. In contrast, infected two-day larvae with Beauveria spp. by $10^{10}$ spore concentration, showed severe destruction of oral hooks, and cuticular papillae surrounding mouth parts. But, control larvae showed normal and intact cuticles, smooth and wellformed body parts, fungal spores on the larval cuticle exhibited severe dehydration, accompanied by cuticular destruction.

In the 14- day post infection pupae, varied mycelia growth stages and numerous fungal spores were observed on magnified surface of infected pupa. No abnormal signs on the pupae longitudinal view were observed. By scanning examination, two-day infected adult flies showed severe com-pound eye destruction with fungal spore aggregation in different parts of compound eye, maxillary bulb, labella and antenna. Spores mushroomed were on adults compound eye $6 \mathrm{hrs}$ post emergence. Germ tubes and fungal spores adhering to ommatidia were clearly seen in $48 \mathrm{hrs}$ post emergence adults. Germ tubes were detected on thorax dorsal surface and legs including tarsus region. Dorsal surface of abdominal region showed a heavy mycelium network with massive aggregations of conidia. In contrast, control flies appeared devoid of any signs of infection like spores or mycelium growth either in head, thorax or abdomen. The detailed were given in figures $(1,2,3,4,5,6,7,8,9,10,11 \& 12)$

\section{Discussion}

Entomopathogenic fungi invaded the host cuticle shortly after its germination, or after limited hyphal growth (Leger et al, 1991). Fungal infection begins when conidia attach to the insect cuticle; the spores germinate, penetrate the insect cuticle and go through the host. Once the fungus penetrated host, it produces toxins and secondary metabolites that overcome the insect immune system to grow rapidly (Roberts, 1981). In the present study, this fact explained the destruction of all stages of $M$. domestica infected by high spore Beauveria spp. concentrations. The sections showed lethal effectiveness on internal and external tissues of the flies. Formation and multiplication of hyphal bodies by Bea-Ouveria spp. inside and outside the host body was marked. In case of $24 \mathrm{hrs}$ postmortem of adults, hyphal bodies and spores were seen in abdominal region near hindgut and vagina. The $72 \mathrm{hrs}$ cadavers showed that the entire body was invaded by fungal spores, whereas the thoracic muscles showed a clear lysis, and the fungal spores obviously attacked the flies' ommatidia. This finding agreed with Toledo et al. (2010) who found that highest concentration of spores and hyphal bodies were detected in compound eyes 
and the abdomen terminal region with lysis of the body fat cells and muscular tissue.

The present SEM showed that conidia of Beauveria spp. attached itself to all body regions of infected flies, particularly areas of dense hair cuticle. This agreed with Boucias et al. (1988), and Boucias et al. (1991). In this case, Beauveria bassiana conidia were abundantly in the hair of infected $4^{\text {th }}$-instar larvae of Velvetbean caterpillar (Anticarsia gemmatalis). Beauveria spp conidia were between ommatidia of compound eye and at articulating membrane of adults' legs. This agreed with Hasaballah, et al. (2017) who recorded B. bassiana and Metarhizium anisopliae conidia on compound eye, thorax, legs and abdomen of $M$. domestica. Toledo et al. (2010) found that conidia of B. bassiana isolate biologically controlled Peregrinus maidi and attacked its compound eyes and legs.

\section{Conclusion}

The present study succeeded in isolating of the entomopathogenic fungus, Beauveria spp. from Musca domestica. SEM and histopathology studies proved that the fungus has destructive effects on external and internal structures of the larvae, pupa and adults of house flies. Thus, fungal spore is recommended for biological control of house flies.

\section{References}

Amaike, S, Keller, NP, 2011: Aspergillus flavus. Ann. Rev. Phytopathol. 49:107-133.

Azzam, S, Hussein, E, 2002: Toxicities of several insecticides to the house fly Musca domestica from different regions in Jordon. Sarhad J. Agric. 18:69-75.

Bancroft, JD, Stevens, A, Turner, DR, 1996: Theory and Practice of Histological Technique. Fourth Ed., Churchill Livingstone, New York, London, San Francisco, Tokyo.

Boucias, DG, Pendland, JC, Latge, JP, 1988: Nonspecific factors involved in attachment of entomopathogenic deuteromycetes to host insect cuticle. Appl. Environ. Microbiol. 54, 7:1795805.

Boucias, DG, Pendland, JC, Latge, JP, 1991: Attachment of mycopathogens to cuticle: The initial event of mycoses in arthropod hosts. In:
Fungal Spore and Disease Initiation in Plants and Animals (GT Cole \& HC Hoch, Eds.).

Farooq, M, Freed, S, 2016: Infectivity of housefly, Musca domestica (Diptera: Muscidae) to different entomopathogenic fungi. Braz. J. Microbiol.47, 4: 807-816.

Förster, M, Klimpel, S, Sievert, K, 2009: The house fly (Musca domestica) as a potential vector of metazoan parasites caught in a pig-pen in Germany. Vet. Parasitol. 160, 1/2:163-7.

Geden, CJ, 2012: Status of bio pesticides for control of house flies. J. Biopesticid. 5:1-8.

Hasaballah, AI, Fouda, MA, Hassan, MI, Om ar, GM, 2017: Pathogenicity of Beauveria bassiana \& Metarhizium anisopliaeon the adult housefly. L. Egypt. Acad. J. Biol. Sci. 10, 5:79-86.

Hafez, M, Osman, MF, Erakey, MA, 1971: Studies on control of house flies in Egypt by chemosterilants. IV. Laboratory evaluation of chemosterilant baits on Musca domestica vicina. J. Econ. Entomol. 64, 4:979-81.

Khan, S, Guo, L, Maimaiti, Y, Mijit, M, Qiu, DW, 2012: Entomopathogenic fungi as microbial biocontrol agent. Mol. Plant Breed. 3:63-79. Lecuona, RE, Turica, M, Tarocco, F, Crespo, D, 2005: Microbial control of Musca domestica (Diptera: Muscidae)with selected strains of $\mathrm{Bea}$ uveria bassiana. J. Med. Entomol. 42, 3: 332-6. Leger, SRJ, 1991: Integumental as a barrier to microbial infections. In: Physiology of Insect Epidermis Retnakaran A, Binnington K, (Eds.). Mwamburi, LA, Laing, MD, Miller, R, 2010: Laboratory screening of insecticidal activities of Beauveria bassiana and Paecilomyceslilacinus against larval and adult house fly (Musca domestica L.). Afri. Entomol. 18, 1:38-46.

Roberts, DW, St Leger, RJ, 2004: Metarhizium spp. cosmopolitan insect-pathogenic fungi: mycological aspects. Adv. Appl. Microbiol. 54, $1: 1-70$.

Roberts, DW, 1981: Toxins of Entomopathogenic Fungi. London: Academic Press

Sales, MDSN, Costa, GLD, Bittencourt, VRE P, 2002: Isolation of fungi in Musca domestica Linnaeus, 1758 (Diptera: Muscidae) captured at two natural breeding grounds in the municipality of Seropédica. Rio de Janeiro. Brazil. Memórias doInstitutoOswaldo Cruz. 97, 8: 1107-10.

Schuster, E, Dunn-Coleman, N, Frisvad, JC, Van Dijck, PW, 2002: On the safety of Aspergillusniger: A review. Appl. Microbiol. Biotechnol. 59, 4/5:426-35.

Seymour, R, Cowgill, MM, Klecka, GM, Ger- 
sich, FM, Mayes, MA, 1984: Occurrence of Aphanomycesdaphiniae infection in laboratory cultures of Daphnia magna. J. Invert. Pathol. 4, 3:109-13

Shah, PA, Pell, JK, 2003: Entomopathogenic fungi as biological control agents. Appl.Microbiol. Biotechnol. 61, 5/6:413-23.

Steinkraus, DC, Geden, CJ, Rutz, DA, Kramer, JP, 1990: First report of the natural occurence of Beauveria bassiana (Moniliales: Moniliaceae) in Musca domestica (Diptera: Muscid- ae). J. Med. Entomol. 27, 3:309-12.

Toledo, AV, Lenicov, AMM, Lastra, C, 2010: Histopathology caused by the entomopathogenic fungi, Beauveria bassiana and Metarhizium anisopliae, in the adult planthopper, Peregrinus maidis, a maize virus vector. J. Insect Sci. 10:1-6. Zarrin, M, Vazirianzadeh, B, Solary, SS, Mahmoudabadi, AZ, Rahdar, M, 2007: Isolation of fungi from housefly (Musca domestica) in Ahwaz, Iran. Pakis. J. Med. Sci. 23, 6:917.

\section{Explanation of figures}

Fig. 1: Histopathology of control and infected larvae. (A): Control larva stained by PAS showed normal structures, cuticular integ ument (ci), muscular system (ms) and cephalophryngeal region (cp) spores. (B): Infected larva stained by PAS showed degenerated tissue (dt) lysis of adipose tissue (at). (C): Infected larva stained by PAS showed fungal spores in outer surface and body cavity (fs). (D): Infected larva stained by MT showed fungal spores in outer surface of larva ( $\mathrm{fs}$ ).

Fig. 2: Histopathology of control and 24hrs postmortem adults. (A): Control female stained by PAS showed normal structures, head (h), thorax (th) and abdomen (ab). (B): Control adult male stained by H\&E showed normal testes (t) and Malpighian tubules (mt). (C): Infected female stained by MT showed aggregation of fungal spores (fs) in abdominal region of vii-ix abdominal segments; illustrate hindgut (hg) and vagina (v). (D): Higher magnification.

Fig. 3: Histopathology of $72 \mathrm{hrs}$ postmortem after death adults. (A): Female stained by MT showed muscular lysis (ly) in thoracic region and fungal spores (fs) in the compound eyes. (B): Female stained by MT showed fungal aggregation (fs) in the compound eyes. (C): Higher magnification of infected adult male stained by MT showing lysis (ly). (D): Higher magnification of female stained by MT showed clear lysis area in muscle of thorax (ly) .

Fig. 4: Histopathology of control and cadaver of 10 days post-infection. (A): Control dead adult of 10 days stained by H\&E showed normal leg (1) and muscle (m). (B): Control dead adult of 10days stained by MT showed normal leg (1) and head region (h). (C): Infected cadaver of 10days stained by MT showed rounded fungal spores (fs) in autolysis tissue (D): Infected cadaver of 10 days stained by PAS showed rounded fungal spores (fs) in proboscis (pr).

Fig. 5: Ten-day cadaver showed fungal spreading in all insect parts.

Fig. 6: Two-days control larva. (A): Mouth part showed normal structure with intact oral hooks (oh) and papillae (p) (X100). (B): Two -days infected larva with Beauveria spp. showed severe destruction (ds) of mouth parts, oral hooks (oh) and papillae (p) (X75).

Fig. 7: Two-day control larva. (A): Inter-segmental region (It) of larvae showed normal and smooth cuticle, and well-formed body parts (X35). (B): Two-day infected larva showed shrunken intersegmental cuticle (It), extreme dehydration and severe destruction (ds) of cuticle with fungal spores (fs) on body surface (X35).

Fig. 8: One-day control M. domestica. (A): whole view of pupa showed normal intersegmental spines (X35).(B): Infected 14-days-pupa with Beauveria spp. showed varied stage of mycelial growth (my) and fungal spores(fs) (X350).

Fig. 9: Control adults of $48 \mathrm{hrs}$ post emergence. (A): head showed normal and intact compound eye (E), maxillary bulb (mb), labella (la) \& antenna (a) (X35). (B): infected adult of $48 \mathrm{hrs}$ post emergence, showed severe destructions(ds) of compound eye(E) \& mouth parts with aggregations of fungal spores(fs) in head different parts (compound eye(E), antenna(a) and labella) (X35).

Fig. 10: Infected adults with Beauveria spp. (A): 6hrs post emergence showed fungal spores (fs) adhering to ommatidia (X750). (B): 48hrs post emergence showed germ tubes (gt) and fungal spores (fs) adhering to ommatidia (X1000).

Fig. 11: Control of $48 \mathrm{hrs}$ post emergence adults. (A): legs (1) no destruction (ds) or mycelium growth (my) (X35). (B): 48hrs post emergence infected adults; showed a network of mycelium (my) and fungal spores (fs) on leg (l) (X150).(C): Germination and penetration of germ tube(gt) on thorax(th) (X1000).

Fig. 12: SEM of control of $48 \mathrm{hrs}$ post emergence adults. (A): abdomen (ab) showing normal cuticle without fungal spores (fs) (X35). 48 hours post emergence infected adult flies. (B): dense network of mycelium (my) on cuticle of abdomen (ab) (X350).

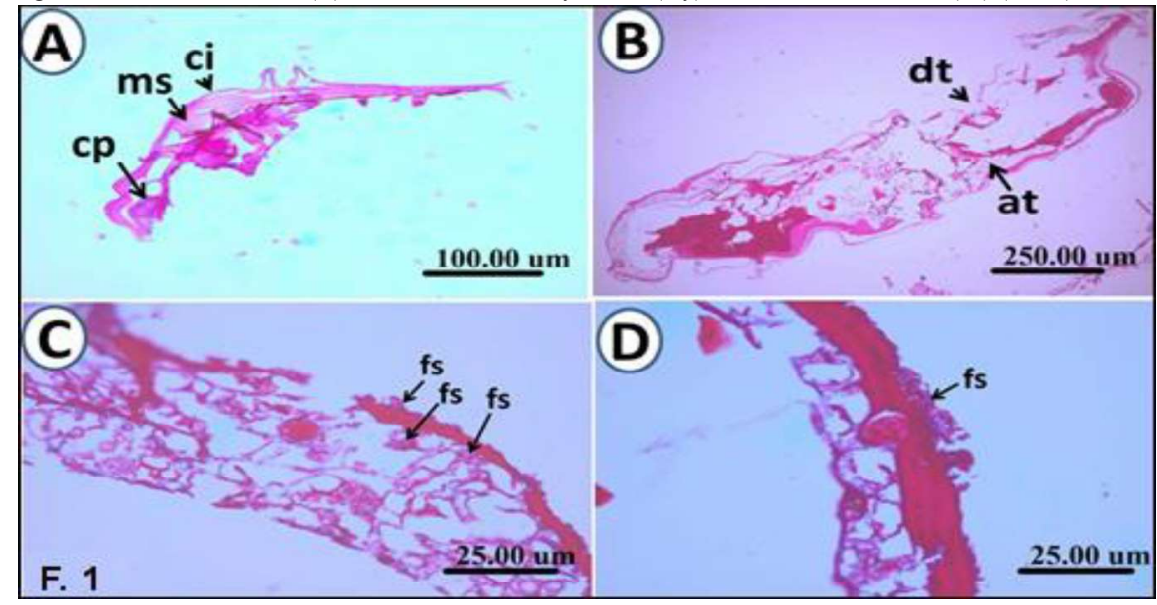



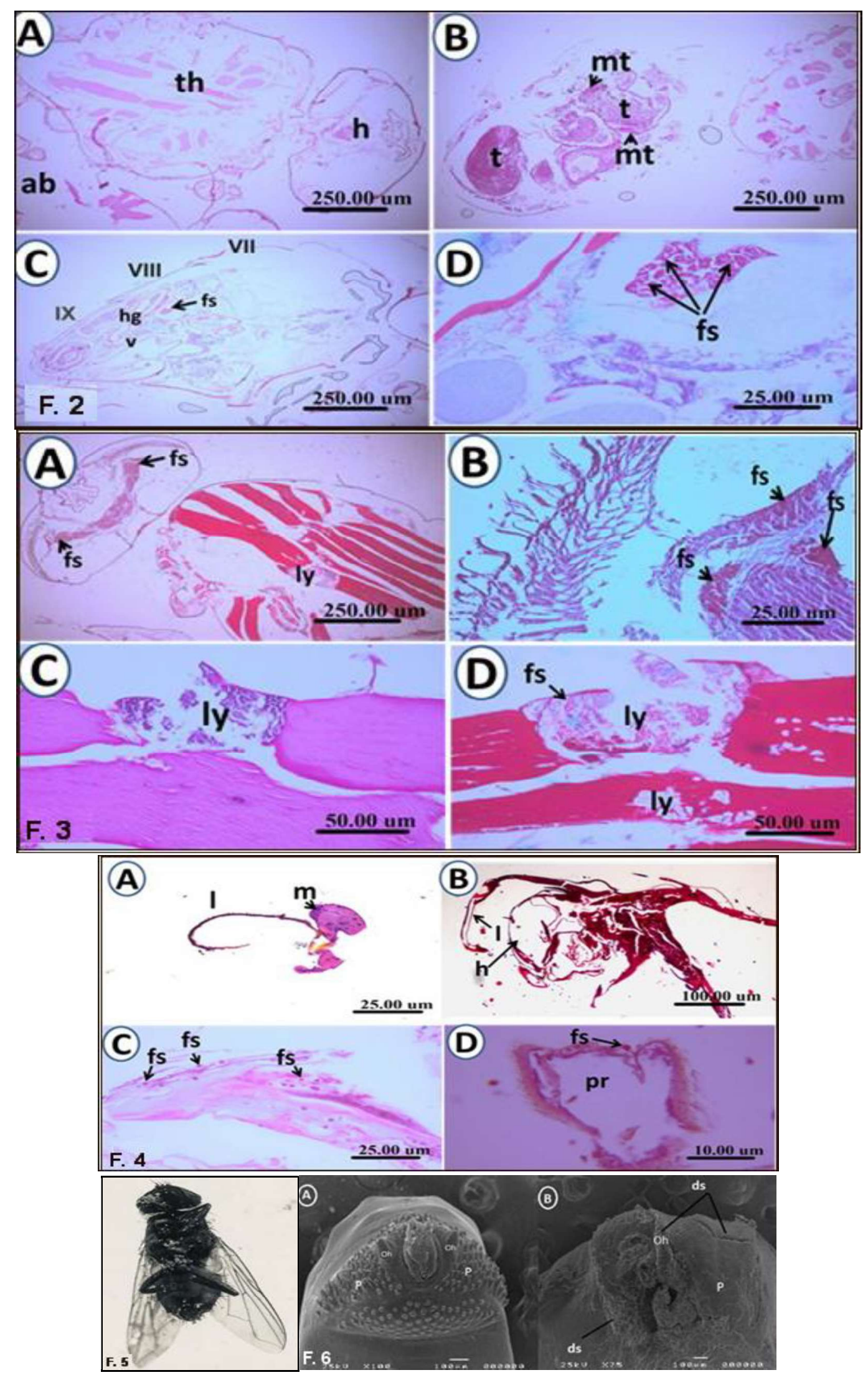


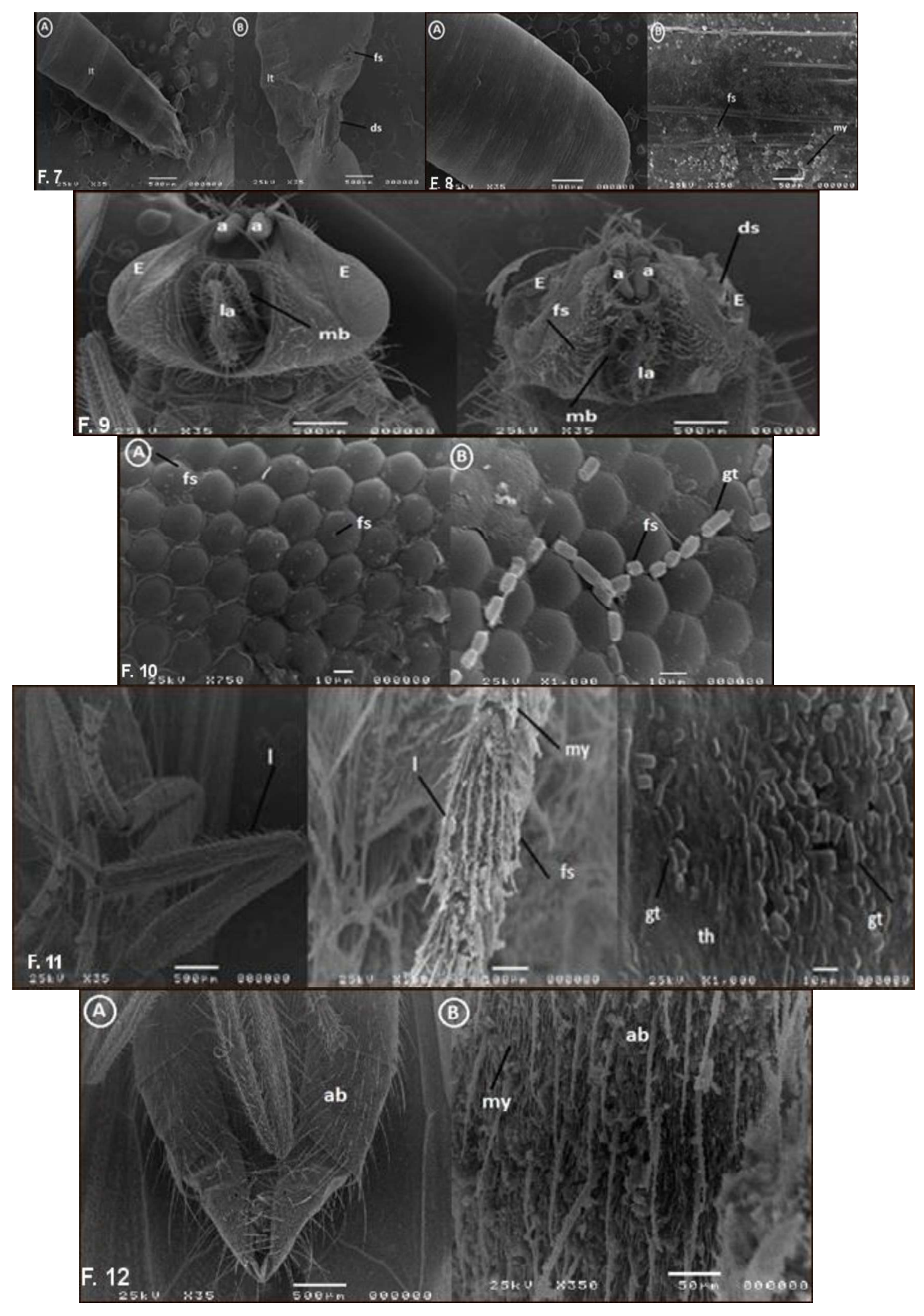

